\title{
Improved density of states Monte Carlo method based on recycling of rejected states
}

\author{
Manan Chopra and J. J. de Pablo ${ }^{\text {a) }}$ \\ Department of Chemical Engineering, University of Wisconsin, Madison, Wisconsin 53706-1691
}

(Received 28 November 2005; accepted 26 January 2006; published online 15 March 2006)

In this paper a new algorithm is presented that improves the efficiency of Wang and Landau algorithm or density of states (DOS) Monte Carlo simulations by employing rejected states. The algorithm is shown to have a performance superior to that of the original Wang-Landau [F. Wang and D. P. Landau, Phys. Rev. Lett. 86, 2050 (2001)] algorithm and the more recent configurational temperature DOS algorithm. The performance of the method is illustrated in the context of results for the Lennard-Jones fluid. (C) 2006 American Institute of Physics. [DOI: 10.1063/1.2178319]

\section{INTRODUCTION}

Markov chain Monte Carlo (MCMC) methods are used extensively to estimate the properties of many-body systems. These methods generate a Markovian random walk in a multidimensional configuration space to calculate the properties of the system of interest. ${ }^{1}$ To calculate the averages with greater statistical accuracy, configurations are visited according to a set of importance weights that are chosen on the basis of some prior knowledge of the contribution of configurations to the averages.

Boltzmann weights are commonly used in the context of statistical physics, but they are not necessarily optimal for a number of important problems in statistical physics, particularly those related to the calculation of free energies; Boltzmann weights assign less importance to configurations having high energies, but such configurations could make substantial contributions to the free energy. Over the years numerous methods have been developed to calculate efficiently free energies from molecular simulations. These methods include umbrella sampling, ${ }^{2}$ multicanonical sampling, ${ }^{3}$ and parallel tempering. ${ }^{4}$ Recently, however, an algorithm introduced by Wang and Landau ${ }^{5}$ has offered a convenient, self-monitoring technique [Wang-Landau density of states (WLDOS)] to calculate the density of states (DOS) of a system, starting from a trivial initial guess.

Over the last few years several improvements to the Wang-Landau method have been proposed. ${ }^{6-9}$ One improvement that we have found to be particularly effective relies on the integration of the configurational temperature ${ }^{10}$ of the system. We refer to this method as configurational temperature density of states (CTDOS) Monte Carlo.

All the above free energy techniques, however, are based on the original rejection scheme of Metropolis et al., ${ }^{11}$ where a random trial displacement from a current state (denoted by $o$ ) to a new state (denoted by $n$ ) is generated with probability $\alpha(o \rightarrow n)$, and is accepted with probability $P_{\text {acc }}(o \rightarrow n)$. The key point is that the rejected states are never included in the

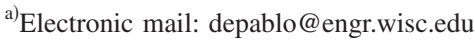

estimation of free energies or, for that matter, in the estimation of any observables. Rejected states are simply discarded and, in some sense, wasted.

Recently, Frenkel has shown that it is possible to include rejected moves in the sampling procedure and improve the statistical accuracy of resulting thermodynamic averages. ${ }^{12}$ Following Frenkel's idea, in this work we show how rejected states can be used to enhance the performance of density of states based simulations.

This paper is organized as follows. First we briefly discuss the detailed balance condition in WLDOS and CTDOS. We then explain how rejected states can be included in WLDOS and CTDOS simulations. After presenting arguments that explain the correctness of the procedure, the performance of the new methods is examined in the context of a simple Lennard-Jones fluid.

\section{REVIEW OF DENSITY OF STATES BASED MONTE CARLO METHODS}

Monte Carlo simulations are generally based on Markov chains designed to obey the condition of detailed balance. That condition ensures that the frequency of transitions from state $o$ onto state $n$ is balanced by the reverse transitions. This property of Markov chains is used extensively in the development of new MCMC algorithms. Note, however, that the condition of detailed balance is only sufficient but not necessary, and alternative simulation schemes that do not satisfy detailed balance can be used to generate an equilibrium ensemble of configurations.

Mathematically, the condition of detailed balance can be stated as

$$
P(o) \mathcal{T}(o \rightarrow n)=P(n) \mathcal{T}(n \rightarrow o),
$$

where $P(s)$ is the probability of state $s$ and $\mathcal{T}(s \rightarrow t)$ denotes the transition probability from state $s$ to state $t$; the probability of the reverse transition is denoted by $\mathcal{T}(t \rightarrow s)$. The transition probabilities that appear in Eq. (1) can be further decomposed into the probability of proposing a transition and accepting that transition, 


$$
P(o) \alpha(o \rightarrow n) P_{\mathrm{acc}}(o \rightarrow n)=P(n) \alpha(n \rightarrow o) P_{\mathrm{acc}}(n \rightarrow o)
$$

If configurations are generated at random, without introducing a bias, then $\alpha(s \rightarrow t)=\alpha(t \rightarrow s)$; substitution into Eq. (2) leads to

$$
\frac{P(n)}{P(o)}=\frac{P_{\mathrm{acc}}(o \rightarrow n)}{P_{\mathrm{acc}}(n \rightarrow o)} .
$$

In WLDOS and CTDOS the aim is to generate flat energy histograms; "energy states" are therefore sampled with a probability inversely proportional to the density of states $\Omega(E)$. Given that in DOS techniques $\Omega$ is calculated "on the fly," in a self-adjusting manner, and given that it is continuously updated throughout the simulation, the condition of detailed balance is only satisfied approximately. After a sufficiently long run, once $\Omega$ becomes approximately invariant, detailed balance is achieved if

$$
\frac{1}{\Omega\left(E_{o}\right)} P_{\mathrm{acc}}(o \rightarrow n)=\frac{1}{\Omega\left(E_{n}\right)} P_{\mathrm{acc}}(n \rightarrow o),
$$

where $\Omega\left(E_{s}\right)$ is the density of states associated with a configuration having energy $E_{s}$. There are various possible choices for $P_{\text {acc }}(s \rightarrow t)$ which satisfy Eq. (4). One possibility is to use the Metropolis acceptance criteria that Wang and Landau used in their original paper,

$$
P_{\text {acc }}(o \rightarrow n)=\min \left\{1, \frac{\Omega\left(E_{o}\right)}{\Omega\left(E_{n}\right)}\right\} \text {. }
$$

Another possible choice for $P_{\text {acc }}$, which also satisfies Eq. (4), is the so-called symmetric rule, ${ }^{13}$

$$
P_{\text {acc }}(o \rightarrow n)=\frac{\Omega\left(E_{o}\right)}{\Omega\left(E_{o}\right)+\Omega\left(E_{n}\right)} .
$$

If configurations are sampled with either of the above acceptance criteria, the resulting Markov chain satisfies detailed balance condition, giving rise to a uniform energy distribution. However, as mentioned above, detailed balance is not a necessary condition; a less stringent "balance" condition can be implemented, which Manousiouthakis and Deem $^{14}$ have shown to be both sufficient and necessary to obtain the sought after equilibrium distribution. By using this balance condition, Frenkel ${ }^{12}$ has formulated an algorithm that uses both rejected and accepted states in canonical Monte Carlo simulations.

We also apply the balance condition but we use it to modify and improve the original WLDOS and CTDOS techniques. In WLDOS, an initial energy range of interest is identified, $E_{\min } \leqslant E \leqslant E_{\max }$, and a random walk is performed in this range. During the random walk, two histograms are updated: one for the density of states, $\Omega(E)$, which represents the current or running estimate, and one for visits to distinct energy states, $h(E)$. Before the simulation begins $h(E)$ is set to zero and $\Omega(E)$ is set to unity. The random walk is performed by choosing an initial state $o$ in the energy range $E_{\min } \leqslant E \leqslant E_{\max }$. Trial moves are then attempted; moves are accepted according to criteria given by Eq. (5). Whenever a trial move is accepted, a histogram entry corresponding to $n$ is incremented according to $h\left(E_{n}\right)=h\left(E_{n}\right)+1$, and $\Omega(E)$ is incremented according to $\Omega\left(E_{n}\right)=\Omega\left(E_{n}\right) f$, where $f$ is an arbitrary convergence factor, that is generally initialized as $e$. If a move is rejected, the new configuration is discarded and the histogram entry corresponding to the old configuration is incremented according to $h\left(E_{o}\right)=h\left(E_{o}\right)+1$; at the same time, the density of states is incremented according to $\Omega\left(E_{o}\right)$ $=\Omega\left(E_{o}\right) f$. This process is repeated until the energy histogram becomes sufficiently flat. When that happens, the energy histogram is reset to zero and the convergence factor is decreased according to $f_{i+1}=\sqrt{f_{i}}$, where $f_{i}$ is the convergence factor corresponding to stage $i$. The process is continued until $f$ becomes sufficiently close to 1 [say, $\left.f<\exp \left(10^{-8}\right)\right]$.

\section{HERETICAL DENSITY OF STATES BASED SAMPLING}

It has been shown that it is possible to construct a sampling scheme in which, for each state that is sampled in a given Markov chain, a subsampling of related states can be performed via an independent Markov web. ${ }^{15}$ These related states can be rejected in conventional sampling. This sampling scheme becomes possible because the equilibrium distribution of all states and the transition rates between all states are known. This procedure has been previously used to enhance the statistical accuracy of "mechanical" observables such as the potential energy. In what follows we present a method to include rejected states in the calculation of entropic quantities such as the density of states.

In order to include rejected states in the WLDOS algorithm, we replace a single WLDOS step between states $o$ and $n$ by $M$, WLDOS steps between them (where $M$ is a very large number). The states $o$ and $n$ will be referred to as microstates and the WLDOS steps as microsteps. These microsteps are analogous to the sampling of "Markovian web" in Ref. 15. If out of $M$ WLDOS steps, $M_{o}$ microsteps are taken to state $o$ (and $M_{n}$ microsteps to state $n$ ), and in every visit to $o$ (or $n) \Omega$ is updated according to $\Omega\left(E_{o}\right)=\Omega\left(E_{o}\right) f[$ or $\left.\Omega\left(E_{n}\right)=\Omega\left(E_{n}\right) f\right]$, then in effect after $M$ microsteps $\Omega\left(E_{o}\right)$ is modified according to $\Omega\left(E_{o}\right)=\Omega\left(E_{o}\right) f^{M_{o}}$ [and $\Omega\left(E_{n}\right)$ according to $\Omega\left(E_{n}\right)=\Omega\left(E_{n}\right) f^{M_{n}}$, respectively]. In the limit of $M$ going to infinity, $M_{o}$ (or $M_{n}$ ) is given by $M_{o}=M \mathcal{P}(o)$ [and $\left.M_{n}=M \mathcal{P}(n)\right]$, where $\mathcal{P}(s)$ is the probability of visiting state $s$ during the microsteps. In other words, $\Omega$ is updated according to $\Omega\left(E_{o}\right)=\Omega\left(E_{o}\right) f^{M^{*} \mathcal{P}(o)}$ [and $\left.\Omega\left(E_{n}\right)=\Omega\left(E_{n}\right) f^{M \mathcal{P}(n)}\right]$. The factor $f^{M}$ can be thought of as a new convergence factor $f_{\text {new }}$; the update equations then become $\Omega\left(E_{o}\right)=\Omega\left(E_{o}\right) f_{\text {new }}^{\mathcal{P}(o)}$ and $\Omega\left(E_{n}\right)=\Omega\left(E_{n}\right) f_{\text {new }}^{\mathcal{P}(n)}$. Since $f$ and $f_{\text {new }}$ are arbitrary constants, for clarity we simply use $f$ (instead of $f_{\text {new }}$ ) for the convergence factor. The probability $\mathcal{P}(s)$ in DOS simulations is proportional to $1 / \Omega\left(E_{s}\right)$; for the microsteps, $\mathcal{P}(o)$ is given by $\Omega(n) /(\Omega(o)+\Omega(n))$ and $\mathcal{P}(n)$ is given by $\Omega(o) /(\Omega(o)$ $+\Omega(n))$. The above step is critical in the working of heretical sampling and is only possible because the $\mathcal{P}(o)$ and $\mathcal{P}(n)$ are known. The transition matrix corresponding to $o$ and $n$ can be calculated as 

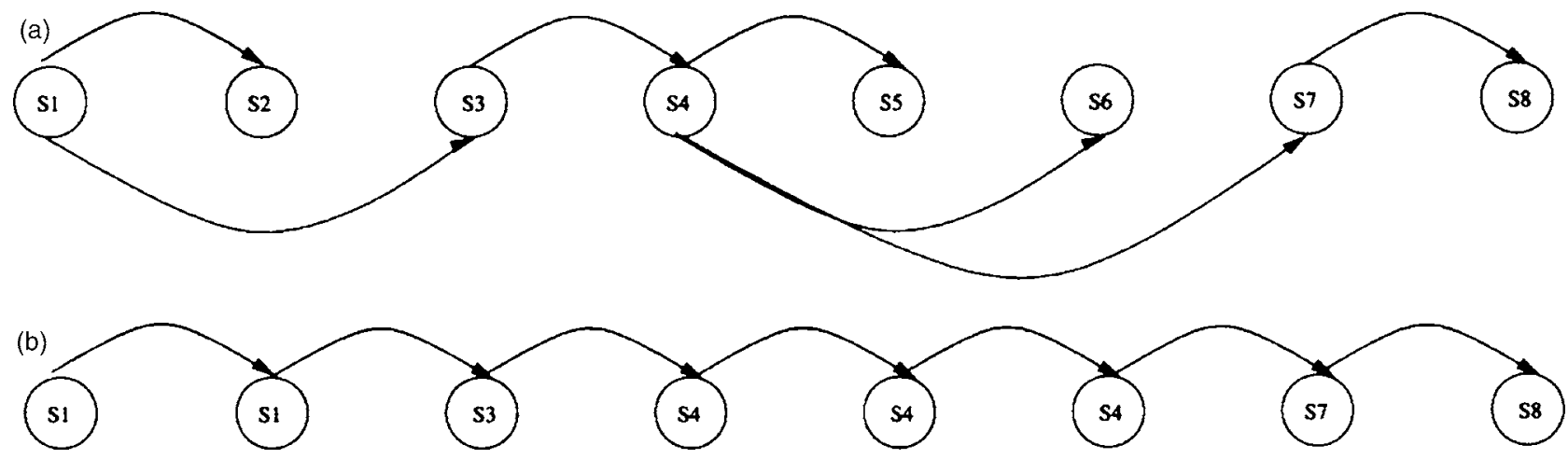

FIG. 1. (a) shows a series of microsteps and microstates. (b) shows a series of macrosteps and macrostates.

$$
\pi=\left[\begin{array}{cc}
\frac{\Omega(o)}{\Omega(o)+\Omega(n)} & \frac{\Omega(n)}{\Omega(o)+\Omega(n)} \\
\frac{\Omega(n)}{\Omega(o)+\Omega(n)} & \frac{\Omega(o)}{\Omega(o)+\Omega(n)}
\end{array}\right]
$$

The transition probabilities in Eq. (7) can be used to estimate modification factors for $\Omega$.

After the microsteps $\Omega$ is updated according to

$$
\Omega(E)= \begin{cases}\Omega\left(E_{o}\right) f^{\left[\Omega\left(E_{n}\right) /\left(\Omega\left(E_{n}\right)+\Omega\left(E_{o}\right)\right)\right]} & \text { if } E=E_{o} \\ \Omega\left(E_{n}\right) f^{\left[\Omega\left(E_{o}\right) /\left(\Omega\left(E_{n}\right)+\Omega\left(E_{o}\right)\right)\right]} & \text { if } E=E_{n} \\ \Omega(E) & \text { if } E \neq E_{o} \text { or } E_{n} .\end{cases}
$$

At the end of the $M$ microsteps, the system is either in state $o$ or in state $n$, with probability $\mathcal{P}(o)$ or $\mathcal{P}(n)$, respectively. The $M$ microsteps can therefore be replaced by a single macrostep from state $o$ to $n$, which is accepted according to Eq. (6).

Figure 1 illustrates the difference between microstates and macrostates with an example where the simulation starts from state $s 1$; a microstate $s 2$ is then generated. State $s 2$ is rejected in the example and hence is not counted in the macrostates. The new macrostate is again $s 1$, from which a new microstate $s 3$ is generated; $s 3$ is accepted and is therefore included in the macrostates. From $s 3$, a microstate $s 4$ is generated and is again accepted. From $s 4$, the first microstate that is generated, $s 5$, is rejected; $s 6$ is generated and also rejected. Finally, $s 7$ is generated and accepted, and is therefore included in the macrostates; from $s 7$, microstate $s 8$ is generated and also accepted, as macrostate. As this example illustrates, microstates include all the configurations generated in the simulation, but macrostates include only the configurations that are accepted in the macrosteps.

The configurations sampled in conventional WLDOS simulations can be viewed as macrosteps; not all the configurations generated in the simulation are used in the estimation of $\Omega$. In the method outlined above, however, all the configurations generated in the simulation are used to estimate $\Omega$ by incorporating them in the microsteps according to Eq. (8). We refer to the new method proposed above as WLDOSH. ${ }^{16}$

The actual algorithmic procedure to implement WLDOSH is outlined below.
(1) As in WLDOS, an initial configuration $o$ is generated in the given energy range $E_{\min } \leqslant E \leqslant E_{\max }$.

(2) A trial state $n$ is then generated.

(3) $\Omega$ is modified according to Eq. (8).

(4) The energy histogram $h(E)$ is not changed during the microsteps.

(5) The move from $o$ to $n$ can be accepted or rejected by using any acceptance criteria that satisfy the detailed balance condition. The symmetric rule of Eq. (6) and the Metropolis rule of Eq. (5) are two examples.

(6) The energy histogram $h(E)$ is updated as in WLDOS during the macrosteps.

Before explaining the procedure to include rejected states in CTDOS simulations, we briefly review the CTDOS algorithm; the details of the method can be found in the original publication. ${ }^{10}$ The temperature of a system is related to its density of states by Boltzmann's equation, ${ }^{17}$

$$
\frac{1}{T}=\left(\frac{\partial S}{\partial E}\right)_{V}=k_{B}\left[\frac{\partial \ln \Omega}{\partial E}\right]_{V},
$$

Eq. (9) can be integrated to determine $\Omega$,

$$
\ln \Omega=\int_{E_{o}}^{E} \frac{1}{k_{B} T} d E .
$$

Equation (10) requires that the temperature be known as a function of energy. Butler et al. ${ }^{18}$ have shown that an intrinsic temperature can be assigned to an arbitrary configuration of a system. This so-called "configurational temperature" is based entirely on configurational information and is given by

$$
\frac{1}{k_{B} T_{\text {config }}}=\frac{\left\langle-\sum_{i} \nabla_{i} \cdot F_{i}\right\rangle}{\left\langle\sum_{i}\left|F_{i}\right|^{2}\right\rangle}=\frac{\mathcal{N}(E)}{\mathcal{D}(E)},
$$

where $F_{i}$ is the force acting on particle $i$.

Just as in WLDOS, in CTDOS a random walk is performed over a specified energy range. During the random walk, two histograms are maintained, one for energy $h(E)$ and one for $\Omega(E)$. Two property accumulators, one for the numerator of Eq. (11), $\mathcal{N}(E)$, and one for the denominator of Eq. (11), $\mathcal{D}(E)$, are also maintained; $T(E)$ is calculated from $\mathcal{N}(E)$, and $\mathcal{D}(E)$ according to $T(E)=\mathcal{N}(E) / \mathcal{D}(E)$. $T(E)$ is later integrated using Eq. (10) to get $\Omega(E)$. In CTDOS, where $\Omega$ is calculated from the ensemble average of the 
$T_{\text {config }}$, the property accumulators have to be modified to include rejected states. We now describe the modification of the accumulator in terms of an arbitrary measurable quantity $\mathcal{F}(E)$. To include rejected states in our sampling procedure, we need to maintain another histogram, $N(E)$, which keeps a track of all the configurations generated in the simulation. If $\mathcal{F}(E)$ has an average $\tilde{\mathcal{F}}(E)$, and we generate two microstates $o$ and $n$, then $\mathcal{F}$ has a value of $\mathcal{F}_{o}$ or $\mathcal{F}_{n}$, respectively. The cumulative value of the accumulator $\mathcal{F}$ is $\tilde{\mathcal{F}}(E) N(E) \tilde{M}(E)$, where $\tilde{M}(E)$ is the average number of visits to an energy state $E$ in the microsteps. Now $M$ regular WLDOS steps are performed between the two states $o$ and $n$; out of a total of $M$ microsteps, $M_{o}$ are to state $o$ and $M_{n}$ are to state $n$. The new cumulative sum gives $\tilde{\mathcal{F}}\left(E_{o}\right) N\left(E_{o}\right) \tilde{M}\left(E_{o}\right)+\mathcal{F}_{o} M_{o}$ and $\tilde{\mathcal{F}}\left(E_{n}\right) N\left(E_{n}\right) \tilde{M}\left(E_{n}\right)+\mathcal{F}_{n} M_{n}$; the new average for $\mathcal{F}(E)$ becomes

$$
\begin{aligned}
& \mathcal{F}\left(E_{o}\right)=\frac{\tilde{\mathcal{F}}\left(E_{o}\right) N\left(E_{o}\right) \tilde{M}\left(E_{o}\right)+\mathcal{F}_{o} M_{o}}{\tilde{M}\left(E_{o}\right) N\left(E_{o}\right)+M_{o}}, \\
& \mathcal{F}\left(E_{n}\right)=\frac{\tilde{\mathcal{F}}\left(E_{n}\right) N\left(E_{n}\right) \tilde{M}\left(E_{n}\right)+\mathcal{F}_{n} M_{n}}{\tilde{M}\left(E_{n}\right) N\left(E_{n}\right)+M_{n}} .
\end{aligned}
$$

The counters $N\left(E_{o}\right)$ and $N\left(E_{n}\right)$ are incremented by unity to register the generation of $o$ and $n$ states. The average $\tilde{M}(E)$ is also modified to include $M_{o}$ and $M_{n}$. Rearranging Eqs. (12) and (13) we have

$$
\begin{aligned}
& \mathcal{F}\left(E_{o}\right)=\frac{\tilde{\mathcal{F}}\left(E_{o}\right) N\left(E_{o}\right)+\mathcal{F}_{o}\left[M_{o} / \tilde{M}\left(E_{o}\right)\right]}{N\left(E_{o}\right)+M_{o} / \tilde{M}\left(E_{o}\right)}, \\
& \mathcal{F}\left(E_{n}\right)=\frac{\tilde{\mathcal{F}}\left(E_{n}\right) N\left(E_{n}\right)+\mathcal{F}_{n}\left[M_{n} / \tilde{M}\left(E_{n}\right)\right]}{N\left(E_{n}\right)+M_{n} / \tilde{M}\left(E_{n}\right)} .
\end{aligned}
$$

In the limit of infinite $M$, and therefore infinitely large $\tilde{M}(E)$, the sample size can be taken as $\tilde{M}(E)$ and $M_{o} / \tilde{M}\left(E_{o}\right)$ $\equiv \mathcal{P}\left(E_{o}\right)$ and $M_{n} / \tilde{M}\left(E_{n}\right) \equiv \mathcal{P}\left(E_{n}\right)$. Substituting the values for
$\mathcal{P}\left(E_{o}\right)$ and $\mathcal{P}\left(E_{n}\right)$ in Eqs. (14) and (15) we arrive at

$$
\begin{aligned}
& \mathcal{F}\left(E_{o}\right)=\frac{\tilde{\mathcal{F}}\left(E_{o}\right) N\left(E_{o}\right)+\mathcal{F}_{o}[\Omega(n) /(\Omega(o)+\Omega(n))]}{N\left(E_{o}\right)+[\Omega(n) /(\Omega(o)+\Omega(n))]}, \\
& \mathcal{F}\left(E_{n}\right)=\frac{\tilde{\mathcal{F}}\left(E_{n}\right) N\left(E_{n}\right)+\mathcal{F}_{n}[\Omega(o) /(\Omega(o)+\Omega(n))]}{N\left(E_{n}\right)+[\Omega(o) /(\Omega(o)+\Omega(n))]} .
\end{aligned}
$$

In order to include rejected states in CTDOS, we need to accumulate $\mathcal{N}$ and $\mathcal{D}$ in the manner described above to calculate $T_{\text {config. }}$. A detailed algorithmic procedure of the proposed new scheme is given below; we refer to this technique as CTDOSH. ${ }^{16}$

(1) As in CTDOS an initial configuration $o$ is generated between the given energy range $E_{\min } \leqslant E \leqslant E_{\max }$.

(2) A trial configuration $n$ is generated.

(3) Then the accumulators $\mathcal{N}(E)$ and $\mathcal{D}(E)$ are updated according to equations below,

$$
\begin{aligned}
& \mathcal{N}\left(E_{o}\right)=\frac{\mathcal{N}\left(E_{o}\right) N\left(E_{o}\right)+\mathcal{N}_{o}[\Omega(n) /(\Omega(o)+\Omega(n))]}{N\left(E_{o}\right)+[\Omega(n) /(\Omega(o)+\Omega(n))]}, \\
& \mathcal{N}\left(E_{n}\right)=\frac{\mathcal{N}\left(E_{n}\right) N\left(E_{n}\right)+\mathcal{N}_{n}[\Omega(o) /(\Omega(o)+\Omega(n))]}{N\left(E_{n}\right)+[\Omega(o) /(\Omega(o)+\Omega(n))]}, \\
& \mathcal{D}\left(E_{o}\right)=\frac{\mathcal{D}\left(E_{o}\right) N\left(E_{o}\right)+\mathcal{D}_{o}[\Omega(n) /(\Omega(o)+\Omega(n))]}{N\left(E_{o}\right)+[\Omega(n) /(\Omega(o)+\Omega(n))]}, \\
& \mathcal{D}\left(E_{n}\right)=\frac{\mathcal{D}\left(E_{n}\right) N\left(E_{n}\right)+\mathcal{D} n[\Omega(o) /(\Omega(o)+\Omega(n))]}{N\left(E_{n}\right)+[\Omega(o) /(\Omega(o)+\Omega(n))]} .
\end{aligned}
$$

(4) The histogram entries for $E_{o}$ and $E_{n}$ in $N(E)$ are incremented by unity.

(5) Again, as in WLDOSH, $h(E)$ is not modified in the microsteps.

(6) The move from $o$ to $n$ is accepted or rejected according to appropriate detailed balance criteria.

(7) As the data for configurational temperature in the initial stages are not very accurate (due to a gross violation of

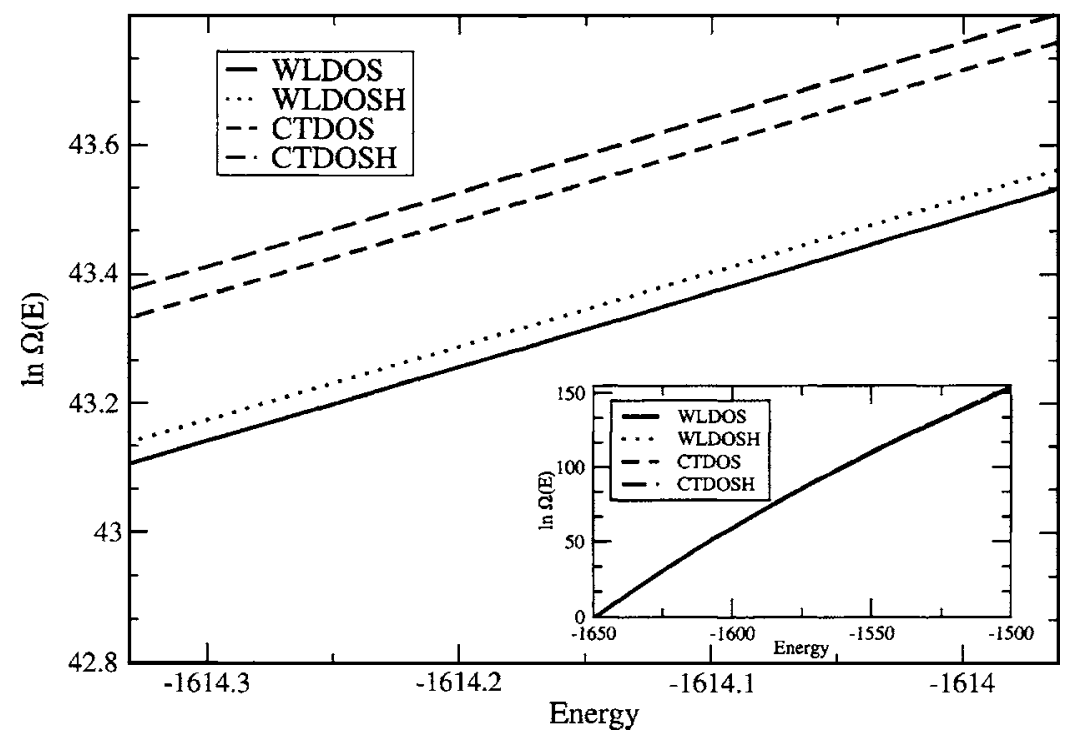

FIG. 2. Expanded scale of the density of states for the LJ fluid calculated by four different techniques, WLDOS (solid), WLDOSH (dot-dot line), CTDOS (dashdash line), CTDOSH (double dash-dash line). The inset shows the density of states calculated by the four techniques over the entire energy range considered in this work. 


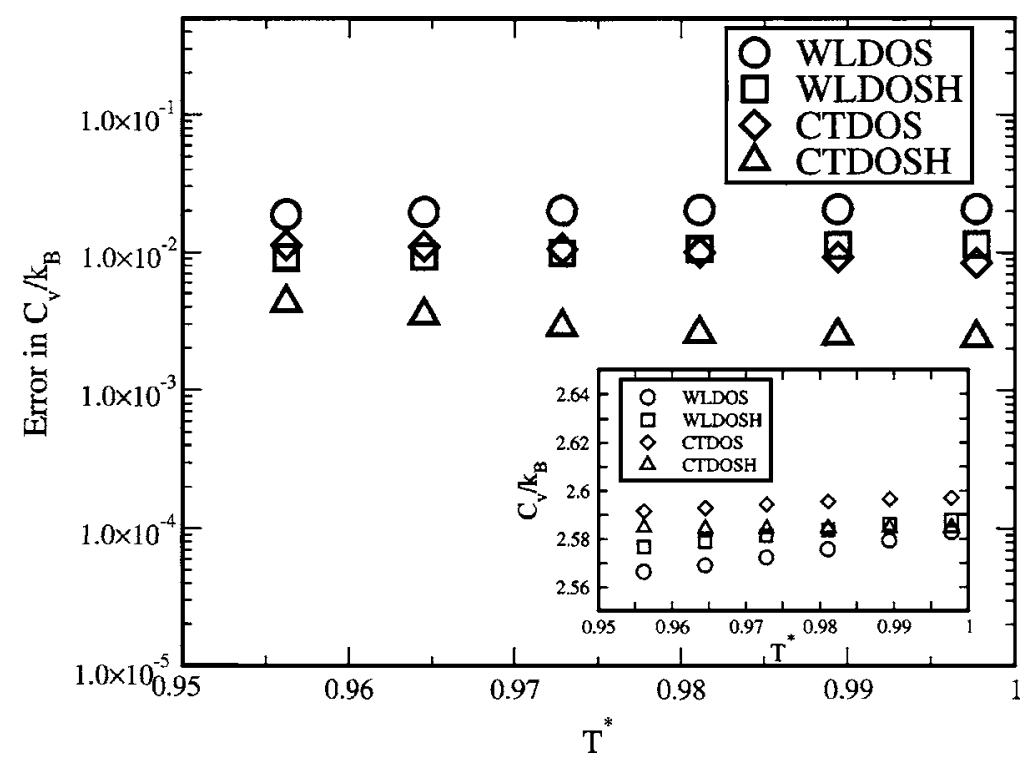

FIG. 3. Statistical error in the specific heat calculated at different temperatures (note that the $y$ axis is in log scale). The inset shows the specific heat values at different temperatures. detailed balance), the histogram corresponding to the configurational temperature is reset in the initial stages of the simulation.

We now address the conditions under which WLDOSH and CTDOSH methods are correct. As mentioned before, WLDOS and CTDOS never satisfy detailed balance exactly. In the later stages of the simulation, detailed balance is satisfied to within a small error. Earl and Deem have shown that for adaptive Monte Carlo techniques such as WLDOS and CTDOS to work detailed balance has to be only satisfied asymptotically. ${ }^{19}$ In the remainder of our discussion we neglect these small violations of detailed balance. The microsteps and macrosteps are performed in WLDOSH and CTDOSH in a predetermined sequence, and these stochastic processes do not satisfy the condition of microscopic reversibility. We therefore show under what conditions they satisfy the weaker balance condition. For a stochastic process to satisfy the balance condition it should be ergodic, it should be Markovian and, finally, it should preserve the invariant distribution.

The first condition is that these stochastic processes should be Markovian. At the level of macrosteps, the movement from one state $o$ to $n$ is a random process having no or limited memory of earlier steps, and we have a Markov chain. Once we have a Markov chain at the level of macrosteps, the balance condition requires that the Markov chain preserve an invariant distribution. As for the macrosteps, we are using the acceptance criteria of Eq. (6), which satisfy detailed balance and are therefore bound to preserve the stationary distribution. Finally, for the systems and moves for which ergodicity can be assumed, the methods, WLDOSH and CTDOSH, satisfy the balance condition of Earl and Deem. The resulting stochastic chain should therefore converge to the correct distribution.

\section{RESULTS FOR LENNARD-JONES FLUID}

In the remainder of this paper we present a numerical comparison of the performance of the WLDOS, CTDOS,
WLDOSH, and CTDOSH algorithms. We refer to the WLDOSH and CTDOSH algorithms collectively as "heretical methods," 16 and to the WLDOS and CTDOS algorithms as "conventional methods." To compare the performance of the above methods, we calculate $\Omega$ for a truncated-and-shifted Lennard-Jones fluid (the potential energy is truncated at $r_{c}$ $=2.5 \sigma)$.

Our simulations were conducted in a cubic box of length $L=6.3 \sigma$, with $N=250$ particles. The dimensionless density of the system is $\rho^{*}=\rho / \sigma^{3}=1.0$ well within the liquid regime. The energy range is set to $-1650 \leqslant E / \epsilon \leqslant-1500$. The simulations are started with a convergence factor $f=e$, and are terminated when it falls below $\exp \left(10^{-10}\right)$. As expected, all methods converge to the same $\Omega$. Figure 2 shows the $\Omega$ estimates from the above four methods.

To measure the performance of the methods we compare the statistical error in the estimates of $\Omega$. For each of the four methods, a set of six independent simulations were conducted, using the same code but different random number seeds. The standard deviation in the specific heat in a set of six simulations for each specific method is taken as a measure of the statistical error for that method. The specific heat was calculated at a range of temperature from $T^{*}=0.95$ to $T^{*}=1.0$. Figure 3 shows the error obtained in specific heats at different temperatures and the inset of Fig. 3 shows the value of specific heat at different temperatures. Table I shows the average statistical error over the whole temperature range in the specific heat for the four techniques. The CPU time invested in the conventional and heretical calculations is the same. There is a small CPU time overhead in configurational

TABLE I. Error in the specific heat $C_{v}$ calculated by WLDOS, CTDOS, WLDOSH, and CTDOSH

\begin{tabular}{cc}
\hline \hline Method & Error \\
\hline WLDOS & 0.0190 \\
WLDOSH & 0.0108 \\
CTDOS & 0.0080 \\
CTDOSH & 0.0027 \\
\hline
\end{tabular}


temperature calculations due to the calculation of Hessians. The errors show that CTDOSH is better than WLDOS by a factor of 50 in terms of statistical accuracy. One important thing must be noted: the efficiency of the schemes presented above depends on the efficiency of the underlying moves used to sample configuration space. The schemes presented above help only in extracting a maximum amount of information from the trial configurations, but do little to improve the decorrelation between successive configurations. For EXEDOS-type simulations, ${ }^{7}$ where a large set of different configurations can correspond to the same value of the order parameter, the above schemes could be even more useful because of the ability to make use of all the generated configurations.

\section{CONCLUSIONS}

We have presented new variants of previously available density of states algorithms that include rejected states in the estimation of $\Omega$. The new methods are useful because they are simple to implement and therefore preserve the elegance of the original Wang-Landau algorithm; with almost negligible computational cost, one can achieve considerable gains in statistical accuracy (for the specific heat a 50 fold increase). This extension is very general in that the rejected states for other types of free energy calculations, such as multicanonical, umbrella sampling, etc., can be also used in the same manner presented here to improve the accuracy of the resulting free energies.

\section{ACKNOWLEDGMENTS}

We would like to thank Dr. Orlando Guzmán, Dr. YengLong Chen, and Clark Miller for helpful discussions. This work was supported by UW-MRSEC on Nanostructured Interfaces.

${ }^{1}$ D. Frenkel and B. Smit, Understanding Molecular Simulations-From Algorithms to Applications (Academic, New York, 1996).

${ }^{2}$ G. M. Torrie and J. P. Valleau, Chem. Phys. Lett. 28, 578 (1974).

${ }^{3}$ B. Berg and T. Neuhaus, Phys. Lett. B 267, 249 (1991).

${ }^{4}$ C. J. Geyer and E. A. Thompson, J. Am. Stat. Assoc. 90, 909 (1995).

${ }^{5}$ F. Wang and D. P. Landau, Phys. Rev. Lett. 86, 2050 (2001).

${ }^{6}$ A. Ethan and J. J. de Pablo, J. Chem. Phys. 122, 124109 (2005).

${ }^{7}$ N. Rathore, Q. L. Yan, and J. J. de Pablo, J. Chem. Phys. 120, 5781 (2004).

${ }^{8}$ E. B. Kim, R. Faller, Q. Yan, N. L. Abbott, and J. J. de Pablo, J. Chem. Phys. 117, 7781 (2002).

${ }^{9}$ P. Dayal, S. Trebst, S. Wessel, D. Wurtz, M. Troyer, S. Sabhapandit, and S. N. Coppersmith, Phys. Rev. Lett. 92, 097201 (2004).

${ }^{10}$ Q. Yan and J. J. de Pablo, Phys. Rev. Lett. 90, 035701 (2003).

${ }^{11}$ N. Metropolis, A. Rosenbluth, M. Rosenbluth, A. Teller, and E. Teller, J. Chem. Phys. 21, 1087 (1953).

${ }^{12}$ D. Frenkel, Proc. Natl. Acad. Sci. U.S.A. 101, 17571 (2004).

${ }^{13}$ A. A. Barker, Aust. J. Phys. 18, 119 (1956).

${ }^{14}$ V. I. Manousiouthakis and M. W. Deem, J. Chem. Phys. 110, 2753 (1999).

${ }^{15}$ G. C. Boulougouris and D. Frenkel, J. Chem. Theory Comput. 1, 389 (2005).

${ }^{16}$ The " $\mathrm{H}$ " in WLDOSH and CTDOSH is inspired by a talk given by Professor Daan Frenkel at the CSCM meeting, 2005 (Erice, Italy) on the subject of recycling of rejected states, which he titled "Heretical Sampling."

${ }^{17}$ D. A. McQuarrie, Statistical Mechanics (Harper Collins, New York, 1976).

${ }^{18}$ B. D. Butler, G. Ayton, O. G. Jepps, and D. J. Evans, J. Chem. Phys. 109, 6519 (1998)

${ }^{19}$ D. J. Earl and W. W. Deem, J. Phys. Chem. B 109, 6701 (2005). 